\title{
Genetic predisposition to obesity leads to increased risk of type 2 diabetes
}

\author{
S. Li • J. H. Zhao • J. Luan • C. Langenberg • \\ R. N. Luben • K. T. Khaw • N. J. Wareham • \\ R. J. F. Loos
}

Received: 21 July 2010 /Accepted: 10 December 2010 /Published online: 26 January 2011

(C) The Author(s) 2011. This article is published with open access at Springerlink.com

\begin{abstract}
Aims/hypothesis Obesity is a major risk factor for type 2 diabetes. Recent genome-wide association (GWA) studies have identified multiple loci robustly associated with BMI and risk of obesity. However, information on their associations with type 2 diabetes is limited. Such information could help increase our understanding of the link between obesity and type 2 diabetes. We examined the associations of 12 obesity susceptibility loci, individually and in combination, with risk of type 2 diabetes in the population-based European Prospective Investigation of Cancer (EPIC) Norfolk cohort.

Methods We genotyped 12 SNPs, identified by GWA studies of BMI, in 20,428 individuals (aged 39-79 years at baseline) with an average follow-up of 12.9 years, during which 729 individuals developed type 2 diabetes. A genetic predisposition score was calculated by adding the BMIincreasing alleles across the 12 SNPs. Associations with incidence of type 2 diabetes were examined by logistic regression models.

Results Of the 12 SNPs, eight showed a trend with increased risk of type 2 diabetes, consistent with their
\end{abstract}

Electronic supplementary material The online version of this article (doi:10.1007/s00125-011-2044-5) contains supplementary material, which is available to authorized users.

S. Li · J. H. Zhao · J. Luan · C. Langenberg · N. J. Wareham •

R. J. F. Loos $(\triangle)$

MRC Epidemiology Unit, Institute of Metabolic Science,

Box 285, Addenbrooke's Hospital, Hills Road,

Cambridge CB2 0QQ, UK

e-mail: ruth.loos@mrc-epid.cam.ac.uk

R. N. Luben $\cdot$ K. T. Khaw

Department of Public Health and Primary Care, Institute of Public Health, University of Cambridge,

Cambridge, UK
BMI-increasing effects. Each additional BMI-increasing allele in the genetic predisposition score was associated with a $4 \%$ increased odds of developing type 2 diabetes (OR 1.041, 95\% CI 1.005-1.078; $p=0.02$ ). Adjustment for BMI completely abolished the association with incident type 2 diabetes (OR 1.003, 95\% CI 0.967-1.039; $p=0.89$ ). Conclusions/interpretation The genetic predisposition to obesity leads to increased risk of developing type 2 diabetes, which is completely mediated by its obesitypredisposing effect.

Keywords Genetic predisposition · Genome-wide association studies - Obesity Type 2 diabetes
Abbreviations
EPIC European Prospective Investigation of Cancer
GWA Genome-wide association
SNP Single nucleotide polymorphism

\section{Introduction}

Type 2 diabetes results from a complex interplay between genetic and environmental factors. Overweight/obesity is a major risk factor for type 2 diabetes. Longitudinal studies have consistently shown that obesity-related traits, such as BMI and waist circumference, are associated with the risk of type 2 diabetes [1-3]. The rapid increase in the prevalence of obesity has contributed to the rising prevalence of type 2 diabetes over the last three decades [4, 5]. However, not all individuals with type 2 diabetes are obese, and vice versa, and it is believed that the genetic architecture of type 2 diabetes in obese individuals is different from that in non-obese individuals $[6,7]$. 
Recent large-scale high-resolution genome-wide association (GWA) studies have identified at least 12 single nucleotide polymorphisms (SNPs) in or near the NEGR1, SEC16B, TMEM18, ETV5, GNPDA2, BDNF, MTCH2, SH2B1, FAIM2, FTO, MC4R and KCTD15 genes, unequivocally associated with BMI [8-12]. Among these, the FTO locus has the largest effect, with each risk allele increasing BMI by $0.40-0.66 \mathrm{~kg} / \mathrm{m}^{2}$ and obesity risk by $30 \%$. Of interest is that the FTO locus was first identified by a GWA study for type 2 diabetes [8]. A more detailed examination showed that the FTO-type 2 diabetes association was mediated by the higher BMI observed in type 2 diabetes cases than in controls as adjustment for BMI abolished the association between FTO and type 2 diabetes [8]. It is not known whether most other recently identified obesity susceptibility loci also increase the risk of type 2 diabetes, and whether such association is independent of, or mediated through, BMI. Such information may increase our understanding of the link between obesity and type 2 diabetes. After all, not all obese individuals have type 2 diabetes and vice versa. Furthermore, the collective contribution of the obesity susceptibility variants to the increased risk of type 2 diabetes has not been studied so far.

Thus, in the current study we examined the associations of the 12 obesity susceptibility loci, individually and in combination, with the risk of developing type 2 diabetes in the European Prospective Investigation of Cancer (EPIC) Norfolk cohort, a population-based cohort with an average follow-up period of 12.9 years.

\section{Methods}

Study participants The EPIC-Norfolk study is a prospective population-based cohort study of 25,631 residents living in the city of Norwich and its nearby areas. At baseline, 21,631 individuals aged 39-79 years, all of white European origin and unrelated, had DNA available for genotyping. Of these, we excluded (1) individuals with missing data for any of the variables studied, including age, sex, BMI, and genotype data for all 12 SNPs $(n=629)$; (2) those with an absolute annual change of BMI greater than $2 \mathrm{~kg} / \mathrm{m}^{2}$ or of waist circumference greater than $7 \mathrm{~cm}$ during a follow-up period of 3-4 years $(n=63)$; and (3) those with missing status of type 2 diabetes at baseline or during follow-up $(n=20)$. Of the remaining sample $(n=20,919), 491$ had type 2 diabetes at baseline.

Full details of the study have been described previously $[13,14]$. In brief, trained nurses measured height in centimetres and weight in kilograms and BMI was calculated as weight in kilograms divided by height in meters squared.

Individuals were ascertained as incident type 2 diabetes cases on 30 November 2008 using multiple data sources: self-report of doctor-diagnosed type 2 diabetes on three follow-up health and lifestyle questionnaires; $\mathrm{HbA}_{1 \mathrm{c}}>7 \%$ at either baseline or follow-up health checks; record linkage that traced each participant for type 2 diabetes diagnosis to general practice registers, regional hospital outpatient diabetes registers, hospital admissions data that linked to type 2 diabetes; and Office of National Statistics mortality data with coding for type 2 diabetes. Criteria for qualifying as a confirmed incident type 2 diabetes case were: (1) confirmation of self-report by another data source; or (2) diagnosis captured by an external source alone, independently of participation in study follow-up questionnaires or visit. A validation study showed that these sources identified $96 \%$ of all known type 2 diabetes cases [15]. In the sample of 20,428 individuals (excluding 491 type 2 diabetes cases at baseline) with an average follow-up period of 12.9 years (median 13.3 years, interquartile range 2.1 years), 729 cases of type 2 diabetes were verified.

Descriptive characteristics at baseline of the study samples, stratified by type 2 diabetes status, are reported in Table 1. The Norfolk, UK, Health District Ethics Committee approved the study and all participants gave their informed written consent.

Genotyping We genotyped SNPs rs3101336, rs10913469, rs6548238, rs7647305, rs10938397, rs925946, rs10838738, rs7498665, rs7132908, rs1121980, rs17782313 and rs368794, in or near the NEGR1, SEC16B, TMEM18, ETV5, GNPDA2, BDNF, MTCH2, SH2B1, FAIM2, FTO, $M C 4 R$ and KCTD15 genes, respectively. These 12 SNPs had previously been found to be unequivocally associated with BMI through large-scale GWA studies [8-12]. SNP information and genotyping methods for the 12 SNPs have been described previously in detail (see Electronic supple-

Table 1 Characteristics of the study cohort at baseline, stratified by type 2 diabetes status

\begin{tabular}{llll}
\hline Variable & Non-case & Incident case & Incident or prevalent case \\
\hline$n$ & 19,699 & 729 & 1,220 \\
Men/women, $n(\%)$ & $9,551 / 10,148(48.5 / 51.5)$ & $450 / 279(61.7 / 38.3)$ & $780 / 440(63.9 / 36.1)$ \\
Age (years), mean \pm SD & $58.6 \pm 9.3$ & $61.8 \pm 8.3$ & $62.7 \pm 8.2$ \\
BMI $\left(\mathrm{kg} / \mathrm{m}^{2}\right)$, mean \pm SD & $26.2 \pm 3.7$ & $29.5 \pm 4.3$ & $28.9 \pm 4.4$ \\
\hline
\end{tabular}


mentary material [ESM] Table 1) [16]. All variants met quality control criteria (call rate $>95 \%$, blind duplicate concordance $>97 \%$ and genotype distributions in HardyWeinberg equilibrium, $p>0.05$ ).

Statistical analyses Genotypes were coded 0,1 or 2 according to the number of BMI-increasing alleles for each SNP, with the BMI-increasing alleles defined by the results reported by recent GWA studies [8-12]. We used a general linear model to examine the effect size of each individual variant on BMI, adjusted for age and sex. A logistic regression model was used to examine the association of BMI (as a continuous trait) and each individual variant with the risk of developing type 2 diabetes during follow-up, with age and sex included in the model as covariates.

To examine the collective contribution of the 12 variants, we calculated a genetic predisposition score for each individual by summing the BMI-increasing alleles across the 12 SNPs. The BMI-increasing alleles were not weighted based on their individual effect sizes as we and others have shown that weighting of risk alleles has no material impact on the results [16, 17]. For individuals with missing genotypes for three or fewer SNPs, missing genotype data were replaced with the average allele count of the respective SNP for the purpose of calculating the genetic predisposition score. Individuals with missing genotypes for more than three SNPs were excluded, which resulted in a total sample of 20,026 individuals (i.e. $97.3 \%$ of the total sample) with a genetic predisposition score. Subsequently, we repeated the analyses with the genetic predisposition score in the same way as we did with each individual SNP.

For associations of individual SNPs or the genetic predisposition score with type 2 diabetes, analyses were further adjusted for BMI to examine whether the associations of individual SNPs or of the genetic predisposition score with type 2 diabetes were mediated by their effects on BMI. Abolishment of association between the SNPs or genetic predisposition with type 2 diabetes after adjustment for BMI would be suggestive of causality between BMI and type 2 diabetes. This determination is typically referred to as instrumental variable analysis or Mendelian randomisation $[18,19]$. To further examine whether the observed effect of individual variants or the genetic predisposition score on incident type 2 diabetes was in agreement with the expected effect based on the effect size of individual SNPs or of the score on BMI and the effect size of BMI on type 2 diabetes, we calculated the expected effect size according to the formula $\beta_{\mathrm{E}}=\beta_{\mathrm{GB}} \times \beta_{\mathrm{BD}}$, where $\beta_{\mathrm{E}}$ is the expected effect size of each individual SNP or the genetic predisposition score on type 2 diabetes $(\operatorname{lnOR}), \beta_{\mathrm{GB}}$ is the effect size of each individual SNP or the score on BMI, and $\beta_{\mathrm{BD}}$ is the effect size of BMI on type 2 diabetes $(\operatorname{lnOR})$; the variance of $\beta_{\mathrm{E}}$ was calculated as $\mathrm{SE}_{\mathrm{GB}}{ }^{2} \times \beta_{\mathrm{BD}}{ }^{2}+\mathrm{SE}_{\mathrm{BD}}{ }^{2} \times \beta_{\mathrm{GB}}{ }^{2}$.
SEs are standard errors for the respective $\beta$ values. Then, $\beta_{\mathrm{E}}$ and the observed $\beta_{\mathrm{O}}$ (lnOR of each individual SNP or the score for type 2 diabetes, adjusted for age and sex) were compared by Student's $t$ test, as previously described by Freathy et al. [20]. We used the same samples to make the comparison for each individual SNP or the genetic predisposition score. Figure 1 illustrates this comparison using the genetic predisposition score as an example. We repeated the analysis using both prevalent type 2 diabetes at baseline and incident type 2 diabetes during follow-up.

We estimated the power that our study had to observe a nominally significant association $(p<0.05)$ of each individual SNP or the genetic predisposition score with type 2 diabetes, based on the expected effect size (and its SE) of each individual SNP or the score on type 2 diabetes. The power was estimated using the following coding: power $=1-\mathrm{CDF}$ ['normal', 1.96-(absolute value of expected effect size/ $\mathrm{SE})$, where $\mathrm{CDF}$ is the cumulative distribution function. We also examined the sex differences in the associations by including an interaction term between sex and SNP (or the score) in the respective models. All analyses were performed using SAS version 9.1 (SAS Institute, Cary, NC, USA).

\section{Results}

The effect sizes of individual SNPs on BMI at baseline ranged from 0.058 to $0.328 \mathrm{~kg} / \mathrm{m}^{2}$, with rs 1121980 in the FTO locus showing the largest effect size and rs7647305 in the ETV5 locus the smallest (Table 2). Each additional BMI-increasing allele in the genetic predisposition score was associated with $0.153(95 \%$ CI $0.129-0.177) \mathrm{kg} / \mathrm{m}^{2}\left(p=5.85 \times 10^{-36}\right)$ increase in BMI.

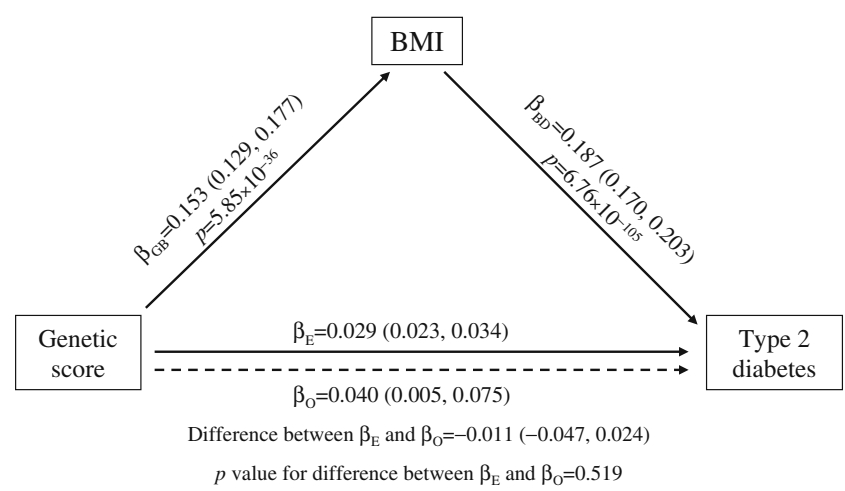

Fig. 1 Illustration of the triangular relationship among the genetic predisposition score, $\mathrm{BMI}$, and type 2 diabetes. $\beta_{\mathrm{GB}}$ represents the effect size of the score on BMI, $\beta_{\mathrm{BD}}$ the effect size of BMI on the risk of developing type 2 diabetes ( $\operatorname{lnOR}$ ), $\beta_{\mathrm{O}}$ the observed effect size of the score on type 2 diabetes, and $\beta_{\mathrm{E}}$ the expected effect size of the score on type 2 diabetes based on $\beta_{\mathrm{GB}}$ and $\beta_{\mathrm{BD}}$. $\beta_{\mathrm{E}}$ and $\beta_{\mathrm{O}}$ were compared by Student's $t$ test. Numbers in parentheses are 95\% CIs 


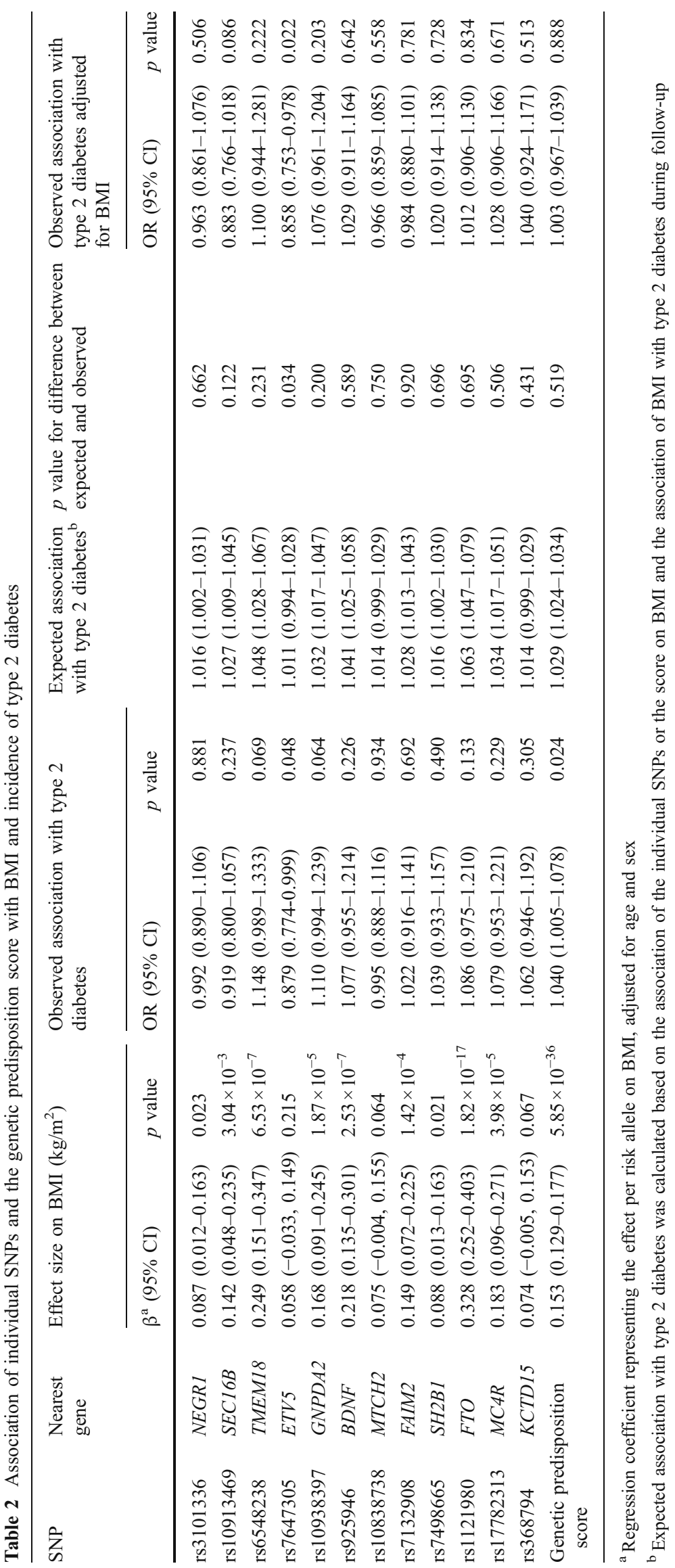


Overall, each unit $\left(\mathrm{kg} / \mathrm{m}^{2}\right)$ increase in BMI was associated with an OR of 1.203 (95\% CI 1.185-1.225, $p=5.62 \times 10^{-108}$ ) for developing type 2 diabetes during follow-up. The odds ratios were similar if both prevalent cases at baseline and incident cases during follow-up were included (OR 1.174, 95\% CI 1.158-1.190, $p=1.44 \times 10^{-116}$ ).

Among the 12 SNPs, eight, in or near TMEM18, GNPDA2, BDNF, FAIM2, SH2B1, FTO, MC4R and $K C T D 15$, did not show an association with type 2 diabetes. However, the effect of these SNPs was directionally consistent as expected from their associations with BMI (OR range 1.039-1.148; Table 2). Further adjustment of these associations for BMI attenuated this positive trend. Four SNPs, in or near NEGR1, SEC16B, ETV5 and $M T C H 2$, tended to be associated with a decreased risk of type 2 diabetes which was directionally opposite to what would be expected based on their BMI-increasing effect (OR range 0.879-0.995). The BMI-increasing allele of rs7647305 in the ETV5 locus was associated with a decreased risk of developing type 2 diabetes $(p=0.048)$. Further adjustment for BMI somewhat strengthened this inverse association (Table 2).

The genetic predisposition score, which considers the collective contribution of the 12 SNPs, showed a positive association with incident type 2 diabetes. Each additional BMI-increasing allele in the genetic predisposition score was associated with a $4.1 \%(95 \%$ CI $0.5-7.8 \%)$ increased odds of developing type 2 diabetes during follow-up (OR $1.041,95 \%$ CI 1.005-1.078; $p=0.02$ ). Adjustment for BMI completely abolished this association (OR 1.003, 95\% CI $0.967-1.039, p=0.89$ ).

The associations of individual SNPs or predisposition score with the incidence of type 2 diabetes were not different from the associations expected based on the observed associations between these SNPs (or the score) and BMI and the association between BMI and type 2 diabetes, except for rs7647305 in the ETV5 locus ( $p=0.02$; Table 2).

Based on the expected effect size of individual SNPs or the genetic predisposition score on type 2 diabetes, we had sufficient power $(>83 \%)$ to observe a nominally significant $(p<0.05)$ association with type 2 diabetes for the genetic predisposition score and for seven SNPs in or near SEC16B, TMEM18, GNPDA2, BDNF, FAIM2, FTO and $M C 4 R$, and reasonable power ( $>62 \%$ ) for two SNPs (near NEGR1 and SH2B1; ESM Table 2).

We repeated the same analyses using both prevalent cases at baseline and incident cases during follow-up (ESM Table 3). The results were largely similar to those of analyses with incident cases only, except that the FTO locus showed a nominal association with an increased risk of type 2 diabetes $(p=0.04)$, whereas the association of the ETV5 locus with type 2 diabetes was no longer observed $(p=0.19)$. Also, the observed and expected associations of SNPs or the score with type 2 diabetes were no longer different $(p \geq 0.15)$.

There were no sex differences in the associations of individual SNPs or the score with type 2 diabetes $(p>0.08$ for all comparisons).

\section{Discussion}

In this large population-based, prospective study with an average follow-up period of 12.9 years, we examined whether the recently identified BMI loci affect the risk of type 2 diabetes independently of, or mediated through, their effects on BMI. Despite the fact that our study was sufficiently powered $(>83 \%)$ for the majority of the SNPs, we did not observe associations of individual SNPs with type 2 diabetes, except for the ETV5 locus, which showed a directionally opposite association with type 2 diabetes $(p=0.048)$. Although the BMI-increasing alleles of most of the SNPs showed a directionally consistent (increasing) trend with the risk of type 2 diabetes, adjustment for BMI attenuated this trend. The overall genetic predisposition to obesity, as estimated by the combination of BMI-increasing alleles across the 12 SNPs, showed an increased risk of developing type 2 diabetes. Each additional risk allele in the genetic predisposition score was associated with a $4 \%$ increased odds for developing type 2 diabetes during follow-up. However, consistent with the individual SNPs analyses, this association was mediated by the effect of the genetic predisposition score on BMI, as adjustment for BMI completely abolished the association. Thus, we observed no marginal effects of the genetic predisposition score on the risk of developing type 2 diabetes and the observed attenuation by BMI was in line with what was expected based on the genetic effects on BMI. Our results support the established causal and positive association between increased BMI and type 2 diabetes.

Information regarding the associations of individual SNPs with type 2 diabetes might improve our understanding of the heterogeneity of the obesity-type 2 diabetes relationship. Such heterogeneity cannot be captured by the genetic predisposition score as it is a composite of genetic variants that might represent a variety of biological pathways. Of the 12 loci examined in this study, only the FTO locus was known to be associated with type 2 diabetes, but this association is fully mediated by FTO's BMI-increasing effect $[8,21]$.

Two GWA studies of BMI reported on the associations between the identified BMI loci and the risk of type 2 diabetes $[11,12]$. In the study by Willer et al. [11], results on the association between eight BMI loci and the risk of type 2 
diabetes were obtained from the DIAGRAM consortium [22]. This meta-analysis of GWA studies of type 2 diabetes case-control studies $\left(n_{\text {cases }}=4,549, n_{\text {controls }}=5,579\right)$ confirmed the association between variation in FTO $(p=6.8 \times$ $10^{-7}$ ) and type 2 diabetes, and also found evidence for the BMI-increasing alleles of variants near TMEM18 $(p=7.6 \times$ $\left.10^{-4}\right)$ and GNPDA2 $\left(p=6.6 \times 10^{-5}\right)$ with an increased risk of type 2 diabetes [22]. These observations seemed consistent with the trends observed in the current study, but a thorough comparison was not possible as no effect sizes or analyses adjusted for BMI have been reported. In addition, no associations between the five other BMI loci (NEGRI, $M T C H 2, S H 2 B 1, M C 4 R$ and KCTD15) were found [22]. Thorleifsson et al. [12] reported on ten BMI loci and the risk of type 2 diabetes $\left(n_{\text {cases }}=3,876, n_{\text {controls }}=28,031\right)$ and confirmed the association between FTO variants and risk of obesity, but, unlike in the DIAGRAM consortium, no association was observed for the TMEM18 locus. The BMIincreasing allele of the FAIM2 locus was found to be associated with type 2 diabetes (OR 1.11, $p=2.3 \times 10^{-4}$ ) and adjustment for BMI only slightly attenuated the association (OR 1.08, $p=0.009$ ) [12]. The FAIM2 variant in our study showed no association with type 2 diabetes, either without (OR 1.02, $p=0.69$ ) or with adjustment (OR 0.98, $p=0.78$ ) for BMI. The BMI-increasing allele of rs7647305 in the ETV5 locus, for which we found suggestive evidence $(p=0.048)$ for a protective effect, was associated with an increased risk of type 2 diabetes $(p=0.04)$ in Thorleifsson et al. [12]. Apart from a weak association $(p=0.04)$ for the $N E G R 1$ variant, none of the other variants (in or near $S E C 16 B, B D N F, S H 2 B 1, M C 4 R$ and KCTD15) studied by Thorleifsson et al. showed association with the risk of type 2 diabetes. In summary, the currently available literature supports a robust association between the BMI-increasing allele of variants in the FTO locus with an increased risk of type 2 diabetes, which is abolished after adjustment for BMI, whereas no associations have been observed for variants in or near SEC16B, BDNF, MTCH2, SH2B1, MC4R and KCTD15. Results for loci in or near NEGR1, TMEM18, ETV5, GNPDA2 and FAIM2 remain inconsistent across studies $[12,22]$, which could, in part, be due to differences in study design (e.g. population-based vs case-control, incident vs prevalent) or environmental factors.

The reason for the lack of overall significant findings for individual SNPs is not clear. Our study had sufficient power to observe a nominally significant association between most SNPs and type 2 diabetes. It can be speculated that the observed SNP-diabetes associations may have been attenuated by other factors, which reduce the power for finding associations in our study. It is also possible that there is true heterogeneity in the biological pathways that underlie the observed SNP-BMI associations, which may explain the lack of associations between obesity SNPs and type 2 diabetes. If such heterogeneity indeed exists, it may explain the lack of associations between obesity SNPs and type 2 diabetes in our study. It may also help explain why some obese individuals do not experience obesity-related metabolic consequences; i.e. they have the 'healthy obesity phenotype' [23-25]. Future large studies or meta-analyses of multiple studies are needed to establish the associations of most of the individual SNPs with type 2 diabetes.

Recent GWA studies of type 2 diabetes have emphasised the importance of beta cell function in the development of type 2 diabetes [26, 27]. However, the association of the genetic predisposition score with type 2 diabetes and its abolishment after adjustment for BMI in the current study also emphasise the importance of obesity in the development of type 2 diabetes and infer causality between the two conditions. Our results also indicate that, in general, genetic variants for obesity do not have an independent effect on the risk of type 2 diabetes.

Our results suggest that the overall genetic susceptibility to obesity, estimated by the genetic predisposition score, increases susceptibility to type 2 diabetes. However, as we have previously shown that the genetic predisposition score explains less than $1 \%$ of the variation in BMI and has limited predictive value for obesity [16], its contribution to variation in type 2 diabetes risk is likely to be similar or even less. Taken together, the results suggest that while the genetic predisposition score has limited clinical value, it can be used as an instrumental variable in the context of Mendelian randomisation.

In conclusion, the genetic predisposition to obesity leads to an increased risk of type 2 diabetes in our large population-based, longitudinal cohort with an average follow-up period of 12.9 years. Our observations suggest a causal relationship between increased BMI/obesity and type 2 diabetes and emphasise the importance of obesity in the development of type 2 diabetes. Larger studies will be required to convincingly establish associations between individual SNPs and type 2 diabetes, and to identify subtle differences between these associations that might enhance our insight into the biological pathways they implicate.

Acknowledgements The European Prospective Investigation of Cancer (EPIC)-Norfolk Study is funded by Cancer Research UK and the Medical Research Council. S. Li is supported by Unilever Corporate Research, UK. We thank A. Bhaniani (Department of Public Health and Primary Care, Strangeways Research Laboratory, University of Cambridge) for his data management support. We also thank our colleagues for their fast and accurate genotyping work in the MRC Epidemiology Unit and colleagues in the EPIC-Norfolk study for data collection over the years. We also wish to thank all participants in this study.

Duality of interest The authors declare that there is no duality of interest associated with this manuscript. 
Open Access This article is distributed under the terms of the Creative Commons Attribution Noncommercial License which permits any noncommercial use, distribution, and reproduction in any medium, provided the original author(s) and source are credited.

\section{References}

1. Vazquez G, Duval S, Jacobs DR Jr, Silventoinen K (2007) Comparison of body mass index, waist circumference, and waist/hip ratio in predicting incident diabetes: a meta-analysis. Epidemiol Rev 29:115-128

2. Colditz GA, Willett WC, Stampfer MJ et al (1990) Weight as a risk factor for clinical diabetes in women. Am J Epidemiol 132:501-513

3. Field AE, Coakley EH, Must A et al (2001) Impact of overweight on the risk of developing common chronic diseases during a 10year period. Arch Intern Med 161:1581-1586

4. Yoon KH, Lee JH, Kim JW et al (2006) Epidemic obesity and type 2 diabetes in Asia. Lancet 368:1681-1688

5. Gregg EW, Cadwell BL, Cheng YJ et al (2004) Trends in the prevalence and ratio of diagnosed to undiagnosed diabetes according to obesity levels in the US. Diab Care 27:2806-2812

6. Timpson NJ, Lindgren CM, Weedon MN et al (2009) Adiposityrelated heterogeneity in patterns of type 2 diabetes susceptibility observed in genome-wide association data. Diabetes 58:505-510

7. Cauchi S, Nead KT, Choquet H et al (2008) The genetic susceptibility to type 2 diabetes may be modulated by obesity status: implications for association studies. BMC Med Genet 9:45

8. Frayling TM, Timpson NJ, Weedon MN et al (2007) A common variant in the FTO gene is associated with body mass index and predisposes to childhood and adult obesity. Science 316:889-894

9. Scuteri A, Sanna S, Chen WM et al (2007) Genome-wide association scan shows genetic variants in the FTO gene are associated with obesity-related traits. PLoS Genet 3:e115

10. Loos RJ, Lindgren CM, Li S et al (2008) Common variants near MC4R are associated with fat mass, weight and risk of obesity. Nat Genet 40:768-775

11. Willer CJ, Speliotes EK, Loos RJ et al (2009) Six new loci associated with body mass index highlight a neuronal influence on body weight regulation. Nat Genet 41:25-34

12. Thorleifsson G, Walters GB, Gudbjartsson DF et al (2009) Genome-wide association yields new sequence variants at seven loci that associate with measures of obesity. Nat Genet 41:18-24
13. Riboli E, Kaaks R (1997) The EPIC project: rationale and study design. European Prospective Investigation into Cancer and Nutrition. Int J Epidemiol 26(Suppl 1):S6-S14

14. Day N, Oakes S, Luben R et al (1999) EPIC-Norfolk: study design and characteristics of the cohort. European Prospective Investigation of Cancer. Br J Cancer 80(Suppl 1):95-103

15. Harding AH, Day NE, Khaw KT et al (2004) Dietary fat and the risk of clinical type 2 diabetes: the European prospective investigation of Cancer-Norfolk study. Am J Epidemiol 159:73-82

16. Li S, Zhao JH, Luan J et al (2010) Cumulative effects and predictive value of common obesity-susceptibility variants identified by genome-wide association studies. Am J Clin Nutr 91:184-190

17. Janssens AC, Moonesinghe R, Yang Q, Steyerberg EW, van Duijn CM, Khoury MJ (2007) The impact of genotype frequencies on the clinical validity of genomic profiling for predicting common chronic diseases. Genet Med 9:528-535

18. Davey Smith G, Ebrahim S (2003) 'Mendelian randomization': can genetic epidemiology contribute to understanding environmental determinants of disease? Int J Epidemiol 32:1-22

19. Lawlor DA, Harbord RM, Sterne JA et al (2008) Mendelian randomization: using genes as instruments for making causal inferences in epidemiology. Stat Med 27:1133-1163

20. Freathy RM, Timpson NJ, Lawlor DA et al (2008) Common variation in the FTO gene alters diabetes-related metabolic traits to the extent expected given its effect on BMI. Diabetes 57:1419 1426

21. Zeggini E, Weedon MN, Lindgren CM et al (2007) Replication of genome-wide association signals in UK samples reveals risk loci for type 2 diabetes. Science 316:1336-1341

22. Zeggini E, Scott LJ, Saxena R et al (2008) Meta-analysis of genome-wide association data and large-scale replication identifies additional susceptibility loci for type 2 diabetes. Nat Genet 40:638-645

23. Sims EA (2001) Are there persons who are obese, but metabolically healthy? Metabolism 50:1499-1504

24. Wildman RP (2009) Healthy obesity. Curr Opin Clin Nutr Metab Care 12:438-443

25. Bluher M (2010) The distinction of metabolically 'healthy' from 'unhealthy' obese individuals. Curr Opin Lipidol 21:38-43

26. McCarthy MI, Hattersley AT (2008) Learning from molecular genetics: novel insights arising from the definition of genes for monogenic and type 2 diabetes. Diabetes 57:2889-2898

27. Perry JR, Frayling TM (2008) New gene variants alter type 2 diabetes risk predominantly through reduced beta-cell function. Curr Opin Clin Nutr Metab Care 11:371-377 